\title{
ALGORITHMIC ASPECTS OF SECURE CONNECTED DOMINATION IN GRAPHS
}

\author{
JaKkePALLi PAVAN KUMAR \\ AND \\ P. Venkata Subba Reddy \\ Department of Computer Science and Engineering \\ National Institute of Technology \\ Warangal, Telangana, India \\ e-mail: jp.nitw@gmail.com \\ pvsr@nitw.ac.in
}

\begin{abstract}
Let $G=(V, E)$ be a simple, undirected and connected graph. A connected dominating set $S \subseteq V$ is a secure connected dominating set of $G$, if for each $u \in V \backslash S$, there exists $v \in S$ such that $(u, v) \in E$ and the set $(S \backslash\{v\}) \cup\{u\}$ is a connected dominating set of $G$. The minimum size of a secure connected dominating set of $G$ denoted by $\gamma_{s c}(G)$, is called the secure connected domination number of $G$. Given a graph $G$ and a positive integer $k$, the Secure Connected Domination (SCDM) problem is to check whether $G$ has a secure connected dominating set of size at most $k$. In this paper, we prove that the SCDM problem is NP-complete for doubly chordal graphs, a subclass of chordal graphs. We investigate the complexity of this problem for some subclasses of bipartite graphs namely, star convex bipartite, comb convex bipartite, chordal bipartite and chain graphs. The Minimum Secure Connected Dominating Set (MSCDS) problem is to find a secure connected dominating set of minimum size in the input graph. We propose a $(\Delta(G)+1)$ approximation algorithm for MSCDS, where $\Delta(G)$ is the maximum degree of the input graph $G$ and prove that MSCDS cannot be approximated within $(1-\epsilon) \ln (|V|)$ for any $\epsilon>0$ unless $N P \subseteq D T I M E\left(|V|^{O(\log \log |V|)}\right)$ even for bipartite graphs. Finally, we show that the MSCDS is APX-complete for graphs with $\Delta(G)=4$.
\end{abstract}

Keywords: secure domination, complexity classes, tree-width, chordal graphs.

2010 Mathematics Subject Classification: 05C69, 68Q25. 


\section{REFERENCES}

[1] P. Alimonti and V. Kann, Some APX-completeness results for cubic graphs, Theoret. Comput. Sci. 237 (2000) 123-134.

https://doi.org/10.1016/S0304-3975(98)00158-3 
[2] A.A. Bertossi, Dominating sets for split and bipartite graphs, Inform. Process. Lett. 19 (1984) 37-40. https://doi.org/10.1016/0020-0190(84)90126-1

[3] A. Brandstädt, V.D. Chepoi and F.F. Dragan, The algorithmic use of hypertree structure and maximum neighbourhood orderings, Discrete Appl. Math. 82 (1998) 43-77. https://doi.org/10.1016/S0166-218X(97)00125-X

[4] A.G. Cabaro, S.R. Canoy Jr and I.S. Aniversario, Secure connected domination in a graph, Int. J. Math. Anal. (Ruse) 8 (2014) 2065-2074. https://doi.org/10.12988/ijma.2014.47221

[5] M. Chlebík and J. Chlebíková, Approximation hardness of dominating set problems in bounded degree graphs, Inform. and Comput. 206 (2008) 1264-1275. https://doi.org/10.1016/j.ic.2008.07.003

[6] E.J. Cockayne, P.J.P. Grobler, W.R. Gründlingh, J. Munganga and J.H. van Vuuren, Protection of a graph, Util. Math. 67 (2005) 19-32.

[7] T.H. Cormen, C.E. Leiserson, R.L. Rivest and C. Stein, Introduction to Algorithms (Prentice Hall, India, 2001).

[8] B. Courcelle, The monadic second-order logic of graphs. I. Recognizable sets of finite graphs, Inform. and Comput. 85 (1990) 12-75. https://doi.org/10.1016/0890-5401(90)90043-H

[9] A.P. De Villiers, Edge Criticality in Secure Graph Domination, Ph.D. Dissertation (Stellenbosch University, 2014).

[10] M.R. Garey and D.S. Johnson, Computers and Intractability: A Guide to the Theory of NP-Completeness (Freeman, New York, 1979).

[11] S. Guha and S. Khuller, Approximation algorithms for connected dominating sets, Lecture Notes in Comput. Sci. 1136 (1996) 179-193. https://doi.org/10.1007/3-540-61680-2_55

[12] T.W. Haynes, S.T. Hedetniemi and P.J. Slater, Fundamentals of Domination in Graphs (Marcel Dekker, Inc., New York, 1998).

[13] T.W. Haynes, S.T. Hedetniemi and P.J. Slater, Domination in Graphs: Advanced Topics (Marcel Dekker, Inc., New York, 1998).

[14] M.A. Henning and A. Pandey, Algorithmic aspects of semitotal domination in graphs, Theoret. Comput. Sci. 766 (2019) 46-57. https://doi.org/10.1016/j.tcs.2018.09.019

[15] W. Jiang, T. Liu, T. Ren and K. Xu, Two hardness results on feedback vertex sets, Lecture Note in Comput. Sci. 6681 (2011) 233-243. https://doi.org/10.1007/978-3-642-21204-8_26

[16] R.M. Karp, Reducibility among combinatorial problems, in: Complexity of Computer Computations (Springer, Boston, 1972) 85-103. https://doi.org/10.1007/978-1-4684-2001-2_9 
[17] H.B. Merouane and M. Chellali, On secure domination in graphs, Inform. Process. Lett. 115 (2015) 786-790. https://doi.org/10.1016/j.ipl.2015.05.006

[18] M. Moscarini, Doubly chordal graphs, Steiner trees, and connected domination, Networks 23 (1993) 59-69.

https://doi.org/10.1002/net.3230230108

[19] H. Müller and A. Brandstädt, The NP-completeness of Steiner tree and dominating set for chordal bipartite graphs, Theoret. Comput. Sci. 53 (1987) 257-265. https://doi.org/10.1016/0304-3975(87)90067-3

[20] B.S. Panda and A. Pandey, Algorithm and hardness results for outer-connected dominating set in graphs, J. Graph Algorithms Appl. 18 (2014) 493-513. https://doi.org/10.7155/jgaa.00334

[21] B.S. Panda, A. Pandey and S. Paul, Algorithmic aspects of b-disjunctive domination in graphs, J. Comb. Optim. 36 (2018) 572-590. https://doi.org/10.1007/s10878-017-0112-6

[22] J. Pavan Kumar, P. Venkata Subba Reddy and S. Arumugam, Algorithmic complexity of secure connected domination in graphs, AKCE Int. J. Graphs Comb. 17 (2020) 1010-1013.

https://doi.org/10.1016/j.akcej.2019.08.012

[23] R. Uehara and Y. Uno, Efficient algorithms for the longest path problem, in: International Symposium on Algorithms and Computation, Lecture Notes in Comput. Sci. 3341 (2004) 871-883. https://doi.org/10.1007/978-3-540-30551-4_74

[24] H. Wang, Y. Zhao and Y. Deng, The complexity of secure domination problem in graphs, Discuss. Math. Graph Theory 38 (2018) 385-396. https://doi.org/10.7151/dmgt.2008

[25] D.B. West, An Introduction to Graph Theory, vol. 2 (Prentice Hall, Upper Saddle River, 2001).

[26] M. Yannakakis, Node-and edge-deletion NP-complete problems, in: Proc. of the Tenth Annual ACM Symposium on Theory of Computing (ACM, New York, 1978) 253-264.

https://doi.org/10.1145/800133.804355

Received 25 March 2019

Revised 17 October 2019

Accepted 19 October 2019 